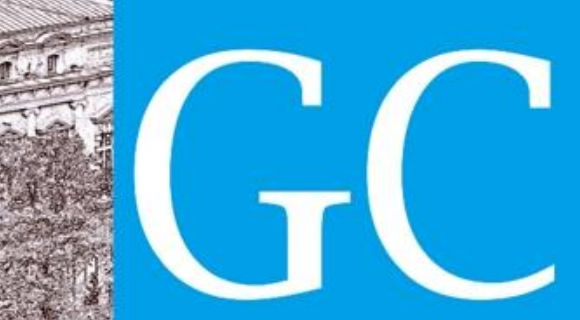

Revista Nacional de Gerenciamento de Cidades

\title{
Consórcios Intermunicipais de Saneamento e de Resíduos Sólidos: elementos para estruturação e consolidação no contexto nacional
}

Intermunicipal Sanitation and Solid Waste Consortia: elements for structuring and consolidation in the national context

Consorcios intermunicipales de saneamiento y desechos sólidos: elementos para a estructuración y consolidación en el contexto nacional

\author{
Katia Sakihama Ventura \\ Professora Doutora, PPGEU-UFSCar, Brasil \\ katiaventura@yahoo.com \\ André Luis Christoforo \\ Professor Doutor, PPGECiv-UFSCar, Brasil \\ christoforoal@yahoo.com.br
}

Ana Beatriz Valim Suquisaqui Mestranda em Engenharia Urbana, PPGEU-UFSCar, Brasil biasuqui@hotmail.com

Kamila Kotsubo Engenheira Civil, UFSCar, Brasil kamila_kotsubo@yahoo.com.br 



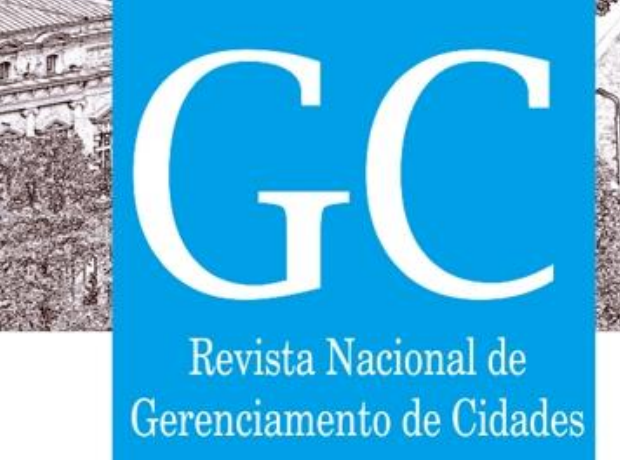

a falta de planejamento de atividades com base no Protocolo de Intenções comparativamente às demandas sociais. Para isto, sugerem i) o estabelecimento de metas e indicadores de avaliação, ii) a busca de parceria, iii) a criação de conselho gestor, iv) a disponibilização de dados e v) a elaboração de plano único de Resíduos Sólidos Urbanos (RSU) para o consórcio.

Os poucos casos identificados na esfera internacional retrataram sobre a constituição de consórcio para valorização da reciclagem de embalagens (CONSORZIO..., 2017) na Itália, a presença de consórcio privado para o serviço de coleta de resíduos sólidos no sudeste asiático (NGOC; SCHNITZER, 2009) e, na Malásia, a forma de garantir recursos financeiros à demanda da gestão de RSU (SAKAWI, 2011). Há um caso que foi constituído entre sete universidades do sul da Inglaterra para gestão dos resíduos gerados por elas (ZHANG et al., 2011).

Estas experiências internacionais ilustram que o funcionamento dos consórcios é orientado pelo alcance de metas e pelo aperfeiçoamento contínuo do processo.

\section{MÉTODOS, TÉCNICAS E INSTRUMENTOS DE PESQUISA}

Este item compreende a apresentação dos procedimentos metodológicos, como sendo levantamento de informações (Fase I), avaliação de consórcios intermunicipais (Fase II) e análise estatística (Fase III).

\subsection{Levantamento de informações (Fase I)}

A busca de dados em endereços eletrônicos de prefeituras e de consórcios brasileiros, bem como a consulta direcionada a este tema em bases científicas (Scientific Electronic Library Online, Periódicos Capes, Web of Science) e técnicas (Observatório dos Consórcios Públicos e Observatório das Metrópoles) representaram a etapa inicial da pesquisa, pois incluiu informações como identificação jurídica, localização e contato do consórcio, assim como, os municípios participantes, relatórios internos e outros documentos institucionais.

Com acesso ao documento desenvolvido por Teixeira e Ventura (2018), que incluiu 86 consórcios nestes eixos temáticos, e a partir da revisão bibliográfica e documental organizada em planilha eletrônica, selecionou-se 26 consórcios para este estudo (Tabela 1).

Tabela 1: Consórcios intermunicipais selecionados no período de 2017 a 2019.

\begin{tabular}{cc}
\hline Macrorregião brasileira & Unidade \\
\hline Sul & 12 \\
Sudeste & 09 \\
Centro-Oeste & 01 \\
Nordeste & 03 \\
Norte & 01 \\
\hline Total & $\mathbf{2 6}$ \\
\hline
\end{tabular}

Fonte: AUTORES, 2020.

Foram realizadas visitas técnicas, em mais de uma vez, a quatro dos consórcios. A existência de casos próximos à instituição de pesquisa (distância até $200 \mathrm{~km}$ ) e a disponibilidade em compartilhar o papel do consórcio com os pesquisadores foram os critérios adotados para referida seleção. 




\begin{tabular}{|c|c|c|c|c|c|c|c|}
\hline \multicolumn{8}{|l|}{$\begin{array}{l}\text { Revista Nacional de } \\
\text { Gerenciamento de Cidades }\end{array}$} \\
\hline & \multicolumn{3}{|c|}{ Setor Administrativo - financeiro - legal (13) } & \multicolumn{4}{|c|}{ Setor Operacional (14) } \\
\hline Variável* & $0(\%)$ & $0,5(\%)$ & $1(\%)$ & Variável* & $0(\%)$ & $0,5(\%)$ & $1(\%)$ \\
\hline EstFin & 53,57 & 0 & 46,43 & MotSoc & 82,14 & 0 & 17,86 \\
\hline Aplnfra & 75,00 & 0 & 25,00 & ElabPro & 0 & 10,71 & 89,29 \\
\hline TerEsta & 96,43 & 0 & 3,57 & CapPes & 25,00 & 25,00 & 50,00 \\
\hline Red\$ & 10,71 & 0 & 89,29 & ACE & 10,71 & 0 & 89,29 \\
\hline Mellnfra & 10,71 & 0 & 89,29 & Resol & 14,29 & 0 & 85,71 \\
\hline AuArt & 46,43 & 0 & 53,57 & CCE & 7,14 & 21,43 & 71,43 \\
\hline ArPro & 71,43 & 0 & 28,57 & DispRS & 46,43 & 14,29 & 39,29 \\
\hline OtrMun & 46,43 & 0 & 53,57 & Comp & 71,43 & 7,14 & 21,43 \\
\hline ProvEsta & 75,00 & 0 & 25,00 & ColRSU & 53,57 & 7,14 & 39,29 \\
\hline Uniao & 57,14 & 0 & 42,86 & LR & 71,43 & 14,29 & 14,29 \\
\hline Aplnter & 96,43 & 0 & 3,57 & AtiSoc & 46,43 & 10,71 & 42,86 \\
\hline RecPriv & 96,43 & 0 & 3,57 & PEV & 82,14 & 0 & 17,86 \\
\hline Admin & 3,57 & 21,43 & 75,00 & ColSel & 64,29 & 0 & 35,71 \\
\hline & & & & $\mathrm{LU}$ & 67,86 & 7,14 & 25,00 \\
\hline
\end{tabular}

*As siglas e identificação das variáveis de caracterização foram apresentadas no Quadro 1. Fonte: AUTORES, 2020.

Cerca de $82 \%$ da amostra (Tabela 2), apresentou duas variáveis com falta de informação que foram Motivação socioambiental (MotSoc) e Criação de locais de entrega voluntária e incentivos a essas práticas (PEV). Os intervalos de confiança da média que permitem estimar a média das informações (Figura 1), com base na média amostral, revelam que os pesos adotados (0.0, 0.5 e 1.0) podem variar, respectivamente, nos intervalos $(38,97 ; 63,41),(2,04 ; 8,28)$ e $(32,42 ; 54,89)$.

Figura 1: Intervalo de confiança e frequência das observações (zero a um), obtidas a partir da Tabela 2, para os consórcios selecionados no período de 2017 a 2019.

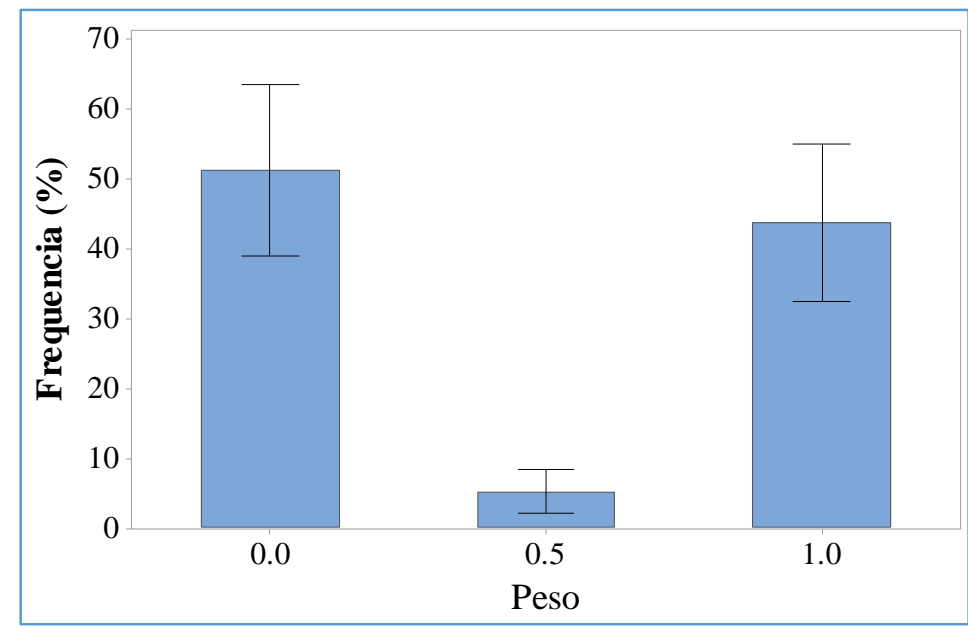

Fonte: AUTORES, 2020.

A Equação 2 apresenta o modelo de regressão multivariada obtida pela análise do objeto de estudo. 


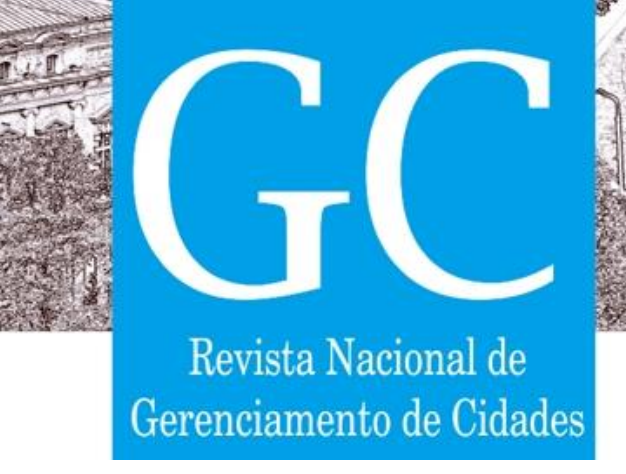

Para futuros estudos, recomenda-se a análise de consórcios intermunicipais com estreita relação a economia circular, cidades inteligentes, tecnologia digital, inclusão social e sustentabilidade para diversificar o foco de abrangência dos consórcios.

\section{Agradecimentos}

O presente trabalho foi realizado com apoio da Coordenação de Aperfeiçoamento de Pessoal de Nível Superior - Brasil (CAPES), sob o código de financiamento 001.

\section{REFERÊNCIAS BILIOGRÁFICAS}

ABRUCIO, F.L.; FILIPPIM, E.S.; DIEGUEZ, R.C. Inovação na cooperação intermunicipal no Brasil: a experiência da Federação Catarinense de Municípios (Fecam) na construção de consórcios públicos. p.1543-1568. Revista Administração Pública, v.47, n.6, 2013.

BARROS, P.M. Consórcio intermunicipal: ferramenta para o desenvolvimento regional. São Paulo: Alga-Oega, 1995. 133p.

BEST, N. J. Cooperação e multi-level governance: o caso do Grande Recife Consórcio de Transporte Metropolitano. Dissertação (mestrado). Escola de Administração de Empresas de São Paulo, Fundação Getulio Vargas, 2011.

BRASIL. Constituição (1988). Constituição da República Federativa do Brasil. Brasília, 1988.

BRASIL. Lei no 11.107 de 6 de abril de 2005. Dispões sobre normas gerais de contratação de consórcios públicos e dá outras providências. Brasília, 2005.

BRASIL. Lei n 12.305 de 2 de agosto de 2010. Institui a Política Nacional de Resíduos Sólidos. Brasília, 2010.

BRASIL. Ministério do Desenvolvimento Regional. Sistema Nacional de Informações sobre Saneamento: Diagnóstico do Manejo de Resíduos Sólidos Urbanos - 2017. Brasília, 2019.

CAÇÃO, R. Testes estatísticos - testes paramétricos e não paramétricos. 43p. 2010. Slides (acervo digital). Disponível em https://pt.slideshare.net/rosariocacao/testes-parametricos-e-nao-parametricos-3396639. Acesso em: 01 mai. 2020.

CALDERAN, T.B. Consórcio público intermunicipal de gerenciamento de resíduos sólidos domésticos: um estudo de caso. Dissertação de mestrado. Lajeado: Programa de Pós-Graduação em Ambiente e Desenvolvimento / UNIVATES 2013. 224P.

CAMPOS, G.M. Estatística Prática para Docentes e Pós-Graduandos - a escolha do teste mais adequado. Material didático. Disponível em: http://www.forp.usp.br/restauradora/gmc/gmc_livro/gmc_livro_cap14.html. Acesso em: 01 mai. 2020.

CHIAVENATO, I. Gestão de Pessoas. 3a edição. Rio de Janeiro: Elsevier, 2010. 579p.

CONSORZIO NAZIONALE IMBALLAGGI (CONAI). Packaging recovery in Italy: the Conai System. 2017. 12p.

CRUZ, M.C.M.T. et at. Consórcios intermunicipais de desenvolvimento: mudando para sobreviver. 2을 Congresso Nacional de Administração Pública, Anais... Brasília: Repositório Digital Saberes em Gestão Pública, 2009. p.1-29.

DALLABRIDA, V.R.; ZIMERMANN, V.J. Descentralização na gestão pública e estruturas subnacionais de gestão do desenvolvimento: o papel dos consórcios intermunicipais. p3-28. Revista Brasileira de Gestão e Desenvolvimento Regional, v. 5, n. 3, p. 3-28, 2009.

FERREIRA, C.F.A.; JUCÁ, J.F.T. Metodologia para avaliação dos consórcios de resíduos sólidos urbanos em Minas Gerais. 


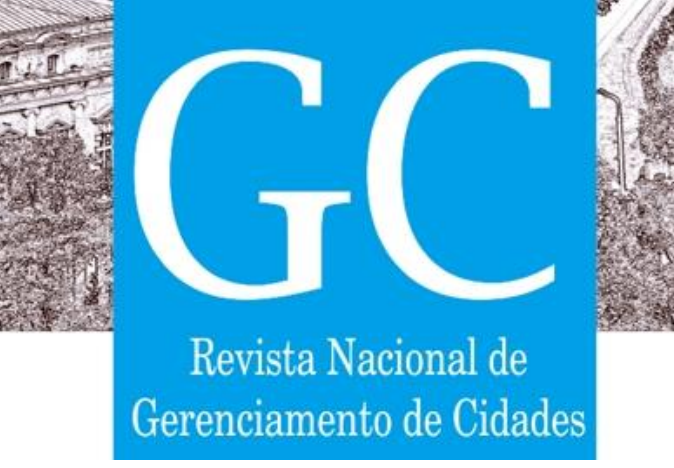

Engenharia Sanitária Ambiental, v.22, n.3, p.513-521, 2017.

FUNASA. Manual de Implantação de Consórcios Públicos de Saneamento. Brasília: FUNASA, 2008. 110p.

FUNASA. Estruturação e Implementação de Consórcios Públicos de Saneamento. Brasília: FUNASA, 2017. 172p.

FUNASA. Cinco passos para implementar um consórcio público de saneamento básico. Teixeira, B.A.N.; Ventura, K.S.; Sassiotto, M.L.P. (orgs). Brasília: Funasa, 2018. 30 p.

GARCIA, M.B.S. et al. Resíduos sólidos: responsabilidade compartilhada. Revista Científica Semioses, v.9, n.2, p.77-91, 2015.

HAIR Jr, J.F. et al. Análise multivariada de dados. 6ạ edição. Porto Alegre: Bookman, 2009. 682p.

IBGE. Perfil de Informações Básicas Municipais. Perfil dos Municípios Brasileiros 2015. Rio de Janeiro: IBGE, 2016. 61p. Disponivel em: https://biblioteca.ibge.gov.br/visualizacao/livros/liv95942.pdf. Acesso em: 01 mai. 2020.

IPEA. Diagnóstico dos Instrumentos Econômicos e Sistemas de Informação para Gestão de Resíduos Sólidos. Relatório de pesquisa. Brasília, 2012. 46p.

JUNQUEIRA, A.T.M. Consórcio intermunicipal: um instrumento de ação. Revista Cepam, v. 1, n. 2, p. 29-36, abr./jun. 1990.

KUMEGAWA, L.S.; VASCONCELOS, M.C.; SILVA, C.L. Consórcio intermunicipal de resíduos sólidos urbanos de Curitiba: uma análise de eficiência. 10p. Anais ... 8o Fórum de Resíduos Sólidos. Curitiba (PR), 2017.

LEOTTI, V. B; BIRCK, A.R.; RIBOLDI, E.J. Comparação dos Testes de Aderência à Normalidade Kolmogorov-Smirnov, AndersonDarling, Cramer-Von Mises e Shapiro-Wilk por Simulação. Anais... In:v11 Simpósio de Estatística Aplicada à Experimentação Agronômica; 50a Reunião Anual da Região Brasileira da Sociedade Internacional de Biometria (RBRAS). Londrina-PR, 2005.

LIMA, A.P.G. Os Consórcios Intermunicipais de Saúde e o Sistema Único de Saúde. p. 985-996. Cadernos de Saúde Pública, v. 16, n. 4, 2000.

LIMA, A. P. G.; PASTRANA, R. M. S. Relatório de pesquisa: diagnóstico da situação atual de consórcios intermunicipais de saúde no Brasil. Brasília: Opas, 2000.

LÖFFLER, E. A gestão da responsabilização nas parcerias intergovernamentais. p.5-34. Revista do Serviço Público, v. 51, n. 2, 2000.

LUCENA, M. D. S. Avaliação de desempenho: métodos e acompanhamento. São Paulo, McGraw-Hill do Brasil, 1997.

MACHADO, J.A.; ANDRADE, M.L.C. Cooperação intergovernamental, consórcios públicos e sistemas de distribuição de custos e benefícios. p.695-720. Revista Administração Pública, v.48, n.3, 2014.

MARRARA, T.; OLIVEIRA, R.M.F. Consórcios para prestação ou regulação de serviços de saneamento básico. p. 457-474. BDM - Boletim de Direito Municipal, ano 33, n. 7, 2017.

MATOS, F.; DIAS, R. A gestão de resíduos sólidos e a formação de consórcios intermunicipais. Revista em Agronegócio e Meio Ambiente, v.4, n.3, p.501-519, 2011.

MATTAR, F. N. Pesquisa de Marketing - execução, análise. 2a edição. São Paulo: Atlas, 1998. 224p.

NGOC, U. N.; SCHNITZER, H. Sustainable solutions for soAceslid waste management in Southeast Asian countries. Waste Management, v. 29, n. 6, p. 1982-1995, 2009.

ÖZTUNA, D.; A. H. ELHAN E E. TÜCCAR. Investigation of Four Different Normality Tests in Terms of Type 1 Error Rate and Power under Different Distributions. Journal of Medicine Cincinnati, v. 36, n. 3, p. 171-176. 2006. 
